\title{
ON THE REPRESENTATION OF CYLINDER FUNCTIONS
}

\author{
ENRICO DE MICHELI
}

\begin{abstract}
In this paper, we present a mixed-type integral-sum representation of the cylinder functions $\mathscr{C}_{\mu}(z)$, which holds for unrestricted complex values of the order $\mu$ and for any complex value of the variable $z$. Particular cases of these representations and some applications, which include the discussion of limiting forms and representations of related functions, are also discussed.
\end{abstract}

\section{INTRODUCTION}

Cylinder functions $\mathscr{C}_{\mu}(z)$ are solutions of the Bessel differential equation

$$
z^{2} \frac{\mathrm{d}^{2} \mathscr{C}_{\mu}}{\mathrm{d} z^{2}}+z \frac{\mathrm{d} \mathscr{C}_{\mu}}{\mathrm{d} z}+\left(z^{2}-\mu^{2}\right) \mathscr{C}_{\mu}=0
$$

where $\mu$ is a fixed complex number. Standard cylinder functions include the Bessel function of the first kind $J_{\mu}(z)$, defined by [10, Eq. 8, p. 40]

$$
J_{\mu}(z) \doteq\left(\frac{z}{2}\right)^{\mu} \sum_{k=0}^{\infty} \frac{(-1)^{k}(z / 2)^{2 k}}{k ! \Gamma(\mu+k+1)} \quad(z \in \mathbb{C}, \mu \in \mathbb{C}, \mu \neq-1,-2, \ldots),
$$

the series on the r.h.s. of (1.2) being convergent absolutely and uniformly on any compact domain of $z \in \mathbb{C}$ and in any bounded domain of $\mu$. The function $J_{\mu}(z)$ is therefore an analytic function of $z$, except for the branch point $z=0$ if $\mu$ is not an integer. The couple of functions $\left\{J_{\mu}(z), J_{-\mu}(z)\right\}$ are linearly independent and their linear combination gives a general solution to (1.1) if $\mu \notin \mathbb{Z}$. When $\mu$ is an integer one is led to introduce the Bessel functions of the second kind $Y_{\mu}(z)$ (also known as Neumann's or Weber's functions), defined by [7, Eq. 10.2.3]

$$
\begin{array}{lr}
Y_{\mu}(z) \doteq \frac{J_{\mu}(z) \cos \mu \pi-J_{-\mu}(z)}{\sin \mu \pi} & \text { if } \quad \mu \notin \mathbb{Z} \\
Y_{m}(z) \doteq \lim _{\mu \rightarrow m} Y_{\mu}(z) & \text { if } \quad m \in \mathbb{Z} .
\end{array}
$$

$\left\{J_{\mu}(z), Y_{\mu}(z)\right\}$ constitutes a linearly independent pair of solutions to equation (1.1) for arbitrary $\mu \in \mathbb{C}$, and therefore the general solution to (1.1) can be written as $\mathscr{C}_{\mu}(z)=c_{1} J_{\mu}(z)+c_{2} Y_{\mu}(z)\left(c_{1}, c_{2}\right.$ constants $)$. Among these combinations, an important role is played by the Bessel functions of the third kind (also known as Hankel's functions) $H_{\mu}^{(1)}(z)$ and $H_{\mu}^{(2)}(z)$, in view of the asymptotic behavior for large $z$, which results to be very useful in applications. They are defined by [7, Eq. 10.4.3]

$$
H_{\mu}^{(1)}(z) \doteq J_{\mu}(z)+\mathrm{i} Y_{\mu}(z) \quad \text { and } \quad H_{\mu}^{(2)}(z) \doteq J_{\mu}(z)-\mathrm{i} Y_{\mu}(z)
$$

2010 Mathematics Subject Classification. 33C10,40C10,33B20.

Key words and phrases. Cylinder functions, Integral representations. 
In the case of purely imaginary values of $z$, ther general solution to (1.1) can be obtained from the functions [7, Eqs. 10.27.6, 10.27.8]

$I_{\mu}(z) \doteq e^{-\mathrm{i} \mu \pi / 2} J_{\mu}(\mathrm{i} z) \quad$ and $\quad K_{\mu}(z) \doteq \begin{cases}\frac{\pi}{2} \mathrm{i}^{\mu+1} H_{\mu}^{(1)}(\mathrm{i} z) & \text { if }-\pi<\arg z \leqslant \frac{\pi}{2} \\ \frac{\pi}{2}(-\mathrm{i})^{\mu+1} H_{\mu}^{(2)}(-\mathrm{i} z) & \text { if }-\frac{\pi}{2}<\arg z \leqslant \pi,\end{cases}$

which are usually referred to as modified Bessel functions. The reader is referred to the classical monograph of G.N. Watson [10] for more information and details on Bessel functions.

Cylinder functions enjoy several integral representations (see, e.g., 10, Chapter $6]$ ), either in terms of definite integrals and of contour integrals. Among the representations of the Bessel function of the first kind $J_{\mu}(z)$ given by integrals on the real line (see, e.g., refs. [7, Chapter 10.9], [3, Sect. 7.3] and [5, Sect. 8.41]), most of them hold only for restricted values of $\mu$. For instance, Poisson-type integral representations [3, Eq. 7.3(3)] hold for Re $\mu>-\frac{1}{2}$, Gubler's representations [3, Eq. 7.3(11)] for $\operatorname{Re} \mu<\frac{1}{2}$, Mehler-Sonine integral formulae hold for $|\operatorname{Re} \mu|<\frac{1}{2}$ [3, Eq. 7.12(12)] or for $|\operatorname{Re} \mu|<1$ [3, Eq. 7.12(14)]. Only the well-known Schläfli's and Heine's representations hold for unrestricted complex values of the index $\mu$. Schläfli's representation [3, Eq. 7.3(9)],

$$
J_{\mu}(z)=\frac{1}{\pi} \int_{0}^{\pi} \cos (z \sin t-\mu t) \mathrm{d} t-\frac{\sin \mu \pi}{\pi} \int_{0}^{\infty} e^{-(z \sinh t+\mu t)} \mathrm{d} t,
$$

holds for $\mu \in \mathbb{C}$, with $\operatorname{Re} z>0$ (it holds also in case $\operatorname{Re} z=0$ provided that $\operatorname{Re} \mu>0$ ), while the two Heine's expressions [3, Eqs. 7.3(31) \& (32)] hold for $\mu \in \mathbb{C}$, but separately in the upper and lower half-plane of the $z$-plane, respectively. Both representations, Schlälli's and Heine's, are made up of the sum of two integrals. From these representations, one can also derive corresponding integral representations of Bessel functions of the second and third kinds by using the relations (1.3) and (1.5).

In Ref. 2] we presented the following integral representation of Fourier-type for the Bessel functions of the first kind $J_{\mu}(z)$ with order $\mu \in \mathbb{C}$, limited to the half-plane $\operatorname{Re} \mu>-\frac{1}{2}$ :

Theorem A ([2, Theorem 1]). Let $\ell \in \mathbb{N}_{0} \doteq\{0,1,2, \ldots\}$ and $\nu$ be any complex number such that $\operatorname{Re} \nu>-\frac{1}{2}$. Then, the following integral representation for the Bessel functions of the first kind $J_{\nu+\ell}(z)$ holds:

$$
J_{\nu+\ell}(z)=(-\mathrm{i})^{\ell} \int_{-\pi}^{\pi} \mathfrak{J}_{z}^{(\nu)}(\theta) e^{\mathrm{i} \ell \theta} \mathrm{d} \theta \quad\left(\ell \in \mathbb{N}_{0}, \operatorname{Re} \nu>-\frac{1}{2}\right),
$$

where the $2 \pi$-periodic function $\mathfrak{J}_{z}^{(\nu)}(\theta)$ is given by

$$
\mathfrak{J}_{z}^{(\nu)}(\theta)=\frac{\mathrm{i}^{\nu}}{2 \pi} e^{\mathrm{i} \nu[\theta-\pi \operatorname{sgn}(\theta)]} e^{\mathrm{i} z \cos \theta} P(\nu,-\mathrm{i} z(1-\cos \theta)),
$$

and $\operatorname{sgn}(\cdot)$ is the sign function, $P(\nu, w) \doteq \gamma(\nu, w) / \Gamma(\nu)$ denotes the normalised incomplete gamma function, $\gamma(\nu, w)$ being the lower incomplete gamma function.

Representation (1.8) can be put in a more appealing form as follows. Any complex number $\mu$ can be represented uniquely as $\mu=\langle\operatorname{Re} \mu\rangle+\{\mu\}$, namely, as the sum of integral and fractional parts, which are defined as follows: $\langle\operatorname{Re} \mu\rangle$ is the nearest 
integer of $\operatorname{Re} \mu$ (sometimes also improperly referred to as the round function of $\operatorname{Re} \mu$ ), with the prescription that, for $n \in \mathbb{Z}$ :

$$
\left\langle n+\frac{1}{2}\right\rangle=n \quad(n \in \mathbb{Z}) .
$$

Accordingly, the (complex) fractional part of $\mu$ is defined as: $\{\mu\} \doteq \mu-\langle\operatorname{Re} \mu\rangle$. Note that this definition implies that in general $\{\mu\} \in \mathbb{C}$ with $-\frac{1}{2}<\operatorname{Re}\{\mu\} \leqslant \frac{1}{2}$.

Let us now consider representation (1.8) and put $\mu=\ell+\nu$ with $\ell \in \mathbb{N}_{0}$ and $\operatorname{Re} \nu>-\frac{1}{2}$. Without loss of generality, we can assume $\langle\operatorname{Re} \nu\rangle=0$. Therefore, $\ell=\langle\operatorname{Re} \mu\rangle$, with the constraint $\langle\operatorname{Re} \mu\rangle \geqslant 0$, and $\nu=\{\mu\}$ is the (complex) fractional part of $\mu$, with $\operatorname{Re}\{\mu\}>-\frac{1}{2}$. Hence, we have $\operatorname{Re} \mu>-\frac{1}{2}$. Thus, with simple calculations formula (1.8) can be re-written as:

$$
J_{\mu}(z)=\frac{\mathrm{i}^{\mu}}{\pi} \int_{0}^{\pi} e^{-\mathrm{i} z \cos \theta} P(\{\mu\},-\mathrm{i} z(1+\cos \theta)) \cos \mu \theta \mathrm{d} \theta \quad\left(\operatorname{Re} \mu>-\frac{1}{2}\right) .
$$

Formula (1.11) generalizes to complex values of $\mu\left(\operatorname{Re} \mu>-\frac{1}{2}\right)$ the classical Bessel integral [7, Eq. 10.9.2]

$$
J_{n}(z)=\frac{\mathrm{i}^{n}}{\pi} \int_{0}^{\pi} e^{-\mathrm{i} z \cos \theta} \cos n \theta \mathrm{d} \theta \quad(n \in \mathbb{Z}),
$$

which holds only for integer values of $n$. The ingredient which allows for this generalization is indeed the (normalised) incomplete gamma function (recall that $P(0, w)=1, w \in \mathbb{C}$ ), whose strong connection with Bessel functions has long been known (see, e.g., [4, 8, 9]). The constraint $\operatorname{Re} \mu>-\frac{1}{2}$, however, prevent us to extend the representation of type (1.11) to other cylinder functions, since these latter always involve values of $J_{\mu}(z)$ with $\operatorname{Re} \mu \leqslant-\frac{1}{2}$ (see (1.3) and (1.5)).

In this paper, we aim indeed at extending the $\mu$-range of validity of formula (1.11) and finding a new representation of $J_{\mu}(z)$ which holds true for unrestricted values of $\mu \in \mathbb{C}$. This representation, which is given is Section 2 (see formula (2.7)), is no longer only integral but it is of mixed-type, an integral plus a finite linear combination of inverse power of $z$. The first part continues to be exactly the integral given in (1.11), whose validity is extented to any $\mu \in \mathbb{C}$, while the finite sum part is actually different from zero only for $\operatorname{Re} \mu \leqslant-\frac{1}{2}$. From this representation, particular values of $\mu$, limiting forms and representation of related functions, i.e. error function and Dawson's integral, are analyzed. The representation of $J_{\mu}(z)$ for any $\mu \in \mathbb{C}$ then makes it possible to obtain a similar representation of mixedtype for the Neumann functions $Y_{\mu}(z), \mu \in \mathbb{C}$. This analysis is given in Section 3 Finally, the results for the Hankel functions $H_{\mu}^{(1,2)}(z)$ are given in Section 4.

\section{Mixed-type integral-Sum Representation of Bessel functions of THE FIRST KIND $J_{\mu}(z)$}

From (1.8) we see that, for fixed $z$ and for $\ell \geqslant 0$, the function $\mathrm{i}^{\ell} J_{\nu+\ell}(z)$ coincides with the $\ell$ th Fourier coefficient of the $2 \pi$-periodic function $\mathfrak{J}_{z}^{(\nu)}(\theta)$. We are then brought to consider the trigonometrical series

$$
\mathfrak{J}_{z}^{(\nu)}(\theta)=\frac{1}{2 \pi} \sum_{\ell=-\infty}^{\infty} \widetilde{\mathfrak{J}}_{\ell}^{(\nu)}(z) e^{-\mathrm{i} \ell \theta},
$$


where $\widetilde{\mathfrak{J}}_{\ell}^{(\nu)}(z)$ denote, for fixed $z$, the $\ell$ th Fourier coefficients of $\mathfrak{J}_{z}^{(\nu)}(\theta)$ :

$$
\widetilde{\mathfrak{J}}_{\ell}^{(\nu)}(z)=\int_{-\pi}^{\pi} \mathfrak{J}_{z}^{(\nu)}(\theta) e^{\mathrm{i} \ell \theta} \mathrm{d} \theta \quad(\ell \in \mathbb{Z}) .
$$

In general, representation (2.1) cannot be written explicitly since the Fourier coefficients with $\ell<0$ are unknown. However, this can be done in two particular cases by exploiting the parity property of $\mathfrak{J}_{z}^{(\nu)}(\theta)$. From equation (1.9) we see that (for fixed $z) \mathfrak{J}_{z}^{(\nu)}(\theta)$ enjoys the symmetry: $\mathfrak{J}_{z}^{(\nu)}(-\theta)=e^{-\mathrm{i} 2 \nu[\theta-\pi \operatorname{sgn}(\theta)]} \mathfrak{J}_{z}^{(\nu)}(\theta)$, which, substituted in (2.2), gives

$$
\widetilde{\mathfrak{J}}_{\ell}^{(\nu)}(z)=\int_{-\pi}^{\pi} \mathfrak{J}_{z}^{(\nu)}(\theta) e^{\mathrm{i} 2 \nu \pi \operatorname{sgn}(\theta)} e^{-\mathrm{i}(\ell+2 \nu) \theta} \mathrm{d} \theta
$$

Formula (2.3) induces thus a $\ell$-index symmetry on the Fourier coefficients $\widetilde{\mathfrak{J}}_{\ell}^{(\nu)}(z)$ only if $2 \nu$ is integer. Precisely, when $\nu \equiv n$ is a nonnegative integral number the symmetry formula reads:

$$
\tilde{\mathfrak{J}}_{\ell}^{(n)}(z)=\widetilde{\mathfrak{J}}_{-\ell-2 n}^{(n)}(z) \quad\left(\ell \in \mathbb{Z} ; n \in \mathbb{N}_{0}\right),
$$

while, when $\nu$ is a nonnegative half-integer: $\nu=n+\frac{1}{2}, n \in \mathbb{N}_{0}$, we have

$$
\widetilde{\mathfrak{J}}_{\ell}^{\left(n+\frac{1}{2}\right)}(z)=-\widetilde{\mathfrak{J}}_{-\ell-2 n-1}^{\left(n+\frac{1}{2}\right)}(z) \quad\left(\ell \in \mathbb{Z} ; n \in \mathbb{N}_{0}\right) .
$$

For a generic complex number $\nu=n+\xi(n \in \mathbb{Z}, \xi \in \mathbb{C})$ an explicit symmetry formula like (2.4) and (2.5) is no longer available. Nevertheless, the Fourier pair (2.1) and (2.2) can be exploited to obtain the extended representation of $J_{\mu}(z)$ we are searching for. To this end, we make use of the following recurrence formula for the normalised incomplete gamma function [7, Eq. 8.8.11]:

$$
P(\xi+n, w)=P(\xi, w)-w^{\xi} e^{-w} \sum_{k=0}^{n-1} \frac{w^{k}}{\Gamma(\xi+k+1)} \quad(\xi \in \mathbb{C} ; n=0,1,2, \ldots)
$$

the sum being obviously understood to be null if $n=0$. Then, the following theorem can be proved.

Theorem 2.1. Let $\mu$ be any complex number. Then, the following representation for the Bessel functions of the first kind $J_{\mu}(z)$ holds:

$$
\begin{aligned}
J_{\mu}(z)= & \frac{\mathrm{i}^{\mu}}{\pi} \int_{0}^{\pi} e^{-\mathrm{i} z \cos \theta} P(\{\mu\},-\mathrm{i} z(1+\cos \theta)) \cos \mu \theta \mathrm{d} \theta \\
& +\frac{\mathrm{i}^{\mu}}{\sqrt{\pi}} e^{\mathrm{i} z} \sum_{j=1}^{-\langle\operatorname{Re} \mu\rangle} \frac{\Gamma\left(j+\mu-\frac{1}{2}\right)}{\Gamma(j) \Gamma(j+2 \mu)}(-2 \mathrm{i} z)^{j+\mu-1} \quad(\mu \in \mathbb{C}),
\end{aligned}
$$

for $z \in \mathbb{C}$ if $\mu \in \mathbb{Z}$, otherwise $z$ belongs to the slit domain $\mathbb{C} \backslash(-\infty, 0]$, and the sum term is understood as zero if $\langle\operatorname{Re} \mu\rangle \geqslant 0$, i.e., if $\operatorname{Re} \mu>-\frac{1}{2}$. 
Proof. Consider the Fourier coefficient $\widetilde{\mathfrak{J}}_{\ell}^{(\nu)}(z)$ (see (2.2) ) with $\ell \in \mathbb{Z}$ and $\nu=n+\xi$, with $\operatorname{Re} \xi>-\frac{1}{2}$ and $n \geqslant 0$ integer. From (2.2) with (1.9) and using (2.6) we have:

$$
\begin{aligned}
\widetilde{\mathfrak{J}}_{\ell}^{(\nu)}(z)= & \frac{\mathrm{i}^{n+\xi}}{2 \pi} \int_{-\pi}^{\pi} e^{\mathrm{i}(n+\xi)[\theta-\pi \operatorname{sgn}(\theta)]} e^{\mathrm{i} z \cos \theta} P(n+\xi,-\mathrm{i} z(1-\cos \theta)) e^{\mathrm{i} \ell \theta} \mathrm{d} \theta \\
= & \frac{(-\mathrm{i})^{n} \mathrm{i} \xi}{2 \pi} \int_{-\pi}^{\pi} e^{\mathrm{i} \xi[\theta-\pi \operatorname{sgn}(\theta)]} e^{\mathrm{i} z \cos \theta}\{P(\xi,-\mathrm{i} z(1-\cos \theta)) \\
& \left.-[-\mathrm{i} z(1-\cos \theta)]^{\xi} e^{\mathrm{i} z(1-\cos \theta)} \sum_{k=0}^{n-1} \frac{[-\mathrm{i} z(1-\cos \theta)]^{k}}{\Gamma(\xi+k+1)}\right\} e^{\mathrm{i}(\ell+n) \theta} \mathrm{d} \theta \\
= & (-\mathrm{i})^{n} \frac{\mathrm{i}^{\xi}}{2 \pi} \int_{-\pi}^{\pi} e^{\mathrm{i} \xi[\theta-\pi \operatorname{sgn}(\theta)]} e^{\mathrm{i} z \cos \theta} P(\xi,-\mathrm{i} z(1-\cos \theta)) e^{\mathrm{i}(\ell+n) \theta} \mathrm{d} \theta \\
& -\frac{(-\mathrm{i})^{n}}{2 \pi} z^{\xi} e^{\mathrm{i} z} \sum_{k=0}^{n-1} \frac{(-\mathrm{i} z)^{k}}{\Gamma(\xi+k+1)} \int_{-\pi}^{\pi} e^{\mathrm{i} \xi[\theta-\pi \operatorname{sgn}(\theta)]}(1-\cos \theta)^{\xi+k} e^{\mathrm{i}(\ell+n) \theta} \mathrm{d} \theta .
\end{aligned}
$$

Now, we assume that $n$ and $\ell$ are such that $n+\ell \geqslant 0$, which amounts to admitting possible negative values of $\ell$. In view of Theorem A, the first term on the r.h.s. of (2.8) is proportional to $\mathrm{i}^{n+\ell} J_{\ell+n+\xi}(z)$ and hence we can write:

$$
\widetilde{\mathfrak{J}}_{\ell}^{(\nu)}(z)=\mathrm{i}^{\ell} J_{\ell+\nu}(z)-\Xi^{(n, \xi, \ell)}(z), \quad\left(\operatorname{Re} \xi>-\frac{1}{2}, n \geqslant 0, \ell+n \geqslant 0\right),
$$

where

$$
\Xi^{(n, \xi, \ell)}(z)= \begin{cases}\frac{(-\mathrm{i})^{n}}{2 \pi} z^{\xi} e^{\mathrm{i} z} \sum_{k=0}^{n-1} \frac{(-\mathrm{i} z)^{k}}{\Gamma(\xi+k+1)} I_{k}^{(n, \xi, \ell)} & \text { if } \quad n \geqslant 1, \\ 0 & \text { if } \quad n=0 .\end{cases}
$$

with

$$
I_{k}^{(n, \xi, \ell)}=\int_{-\pi}^{\pi} e^{\mathrm{i} \xi[\theta-\pi \operatorname{sgn}(\theta)]}(1-\cos \theta)^{\xi+k} e^{\mathrm{i}(\ell+n) \theta} \mathrm{d} \theta, \quad(n \geqslant 1,0 \leqslant k \leqslant n-1 ; \ell+n \geqslant 0) .
$$

The case $n=0$ is trivial since formula (2.9) gives again the result of Theorem A. Then, from now on we assume $n \geqslant 1$. With simple algebraic manipulations the integral $I_{k}^{(n, \xi, \ell)}$ can be written as follows with $0 \leqslant k \leqslant n-1$ and $\operatorname{Re} \xi>-\frac{1}{2}$ :

$$
\begin{aligned}
I_{k}^{(n, \xi, \ell)} & =2(-1)^{n+\ell} \int_{0}^{\pi}(1+\cos \theta)^{\xi+k} \cos (\ell+n+\xi) \theta \mathrm{d} \theta \\
& =\frac{2 \pi(-1)^{n+\ell}}{2^{\xi+k}} \frac{\Gamma(1+2(\xi+k))}{\Gamma(1+k-\ell-n) \Gamma(1+k+n+\ell+2 \xi)},
\end{aligned}
$$

where we used the formula

$$
\int_{0}^{\pi}(1+\cos \theta)^{a} \cos (b \theta) \mathrm{d} \theta=\frac{\pi}{2^{a}} \frac{\Gamma(1+2 a)}{\Gamma(1+a-b) \Gamma(1+a+b)}, \quad\left(\operatorname{Re} a>-\frac{1}{2}\right) .
$$

From (2.10), (2.12), using the Legendre duplication formula for the gamma function and re-indexing the sum setting $j=k-n$, we obtain:

$$
\Xi^{(n, \xi, \ell)}(z)=\frac{(-1)^{\ell}}{\sqrt{\pi}}(2 z)^{n+\xi} e^{\mathrm{i} z} \sum_{j=-n}^{-1} \frac{\Gamma\left(j+n+\xi+\frac{1}{2}\right)(-2 \mathrm{i} z)^{j}}{\Gamma(j+1-\ell) \Gamma(j+1+\ell+2 n+2 \xi)},
$$


for $n \geqslant 1$ and $\Xi^{(0, \xi, \ell)}(z)=0$. When $n \geqslant 1$, the condition $n+\ell \geqslant 0$ means that we are considering even the case of Fourier coefficients with negative values of $\ell,-n \leqslant \ell \leqslant-1$, which were precluded in Theorem A. In view of the factor $\Gamma^{-1}(j+1-\ell)$, the terms of the sum in (2.14) are different from zero only if $j \geqslant \ell$. The latter condition, along with $\ell \geqslant-n$, implies that the sum in (2.14) starts from $j=\ell$. Finally, recalling that $\nu=n+\xi$, we obtain for values of $\nu$ such that $\langle\operatorname{Re} \nu\rangle \geqslant-\ell$ and $\operatorname{Re}\{\nu\}>-\frac{1}{2}$ :

$$
\Xi^{(n, \xi, \ell)}(z)= \begin{cases}\frac{(-1)^{\ell}}{\sqrt{\pi}}(2 z)^{\nu} e^{\mathrm{i} z} \sum_{j=\ell}^{-1} \frac{\Gamma\left(j+\nu+\frac{1}{2}\right)(-2 \mathrm{i} z)^{j}}{\Gamma(j+1-\ell) \Gamma(j+1+\ell+2 \nu)} & \text { if } \quad \ell \leqslant-1, \\ 0 & \text { if } \quad \ell \geqslant 0 .\end{cases}
$$

We can now substitute (2.15) into (2.9) to get:

$$
J_{\ell+\nu}(z)=(-\mathrm{i})^{\ell} \widetilde{\mathfrak{J}}_{\ell}^{(\nu)}(z)+\frac{\mathrm{i}^{\ell}}{\sqrt{\pi}}(2 z)^{\nu} e^{\mathrm{i} z} \sum_{j=\ell}^{-1} \frac{\Gamma\left(j+\nu+\frac{1}{2}\right)(-2 \mathrm{i} z)^{j}}{\Gamma(j+1-\ell) \Gamma(j+1+\ell+2 \nu)}
$$

the second term being null for $\ell \geqslant 0$. Given $\ell \leqslant-1$, formula (2.16) holds true only for $\langle\operatorname{Re} \nu\rangle \geqslant-\ell$ and $\operatorname{Re}\{\nu\}>-\frac{1}{2}$. However, for any $\ell \in \mathbb{Z}$ the $\nu$-domain of validity of representation (2.16) can be analytically extended from the half-plane $\operatorname{Re} \nu>-\ell-\frac{1}{2}$ to the half-plane $\operatorname{Re} \nu>-\frac{1}{2}$ if the r.h.s. of (2.16) is proved to be an analytic function which is locally bounded in every compact subset of $\operatorname{Re} \nu>-\frac{1}{2}$. Let see that this is the case indeed.

For what concerns the summation in (2.16), poles can appear from the factor $\Gamma\left(j+\nu+\frac{1}{2}\right)$ only if $\nu$ is a half-integer, $\nu=n+\frac{1}{2}$ and, consequently, when $j+n+1 \leqslant 0$. This implies that we have simple poles when $n \leqslant-\ell-1$. However, for these values of $n$, poles are correspondingly present in the denominator from the term $\Gamma(j+\ell+2 n+2)$, which has simple poles when $2 \ell+2 n+2 \leqslant 0$, that is, if $n \leqslant-\ell-1$, indeed. The ratio between these two gamma terms remains therefore finite.

Let us now consider the term $\widetilde{\mathfrak{J}}_{\ell}^{(\nu)}(z)$ in the case $\ell \leqslant-1$, which reads (see (2.2) and $(1.9)$ ):

$$
\widetilde{\mathfrak{J}}_{\ell}^{(\nu)}(z)=\frac{\mathrm{i}^{\nu}}{2 \pi} \int_{-\pi}^{\pi} e^{\mathrm{i} \nu[\theta-\pi \operatorname{sgn}(\theta)]} e^{\mathrm{i} z \cos \theta} P(\nu,-\mathrm{i} z(1-\cos \theta)) e^{\mathrm{i} \ell \theta} \mathrm{d} \theta .
$$

First, recall that $P(\nu, w)=w^{\nu} \gamma^{*}(\nu, w)$, where:

$$
\gamma^{*}(\nu, w)=\frac{1}{\Gamma(\nu)} \sum_{m=0}^{\infty} \frac{(-w)^{m}}{(\nu+m) \cdot m !} \quad(\nu \neq 0,-1,-2, \ldots),
$$

is a function entire in both $\nu$ and $w$, and $\gamma^{*}(-n, w)=w^{n}$ for $n \in \mathbb{N}_{0}[8$. Then, for $\theta \in(-\pi, \pi]$, it is easy to see these simple bounds hold true: $\left|e^{\mathrm{i} \nu[\theta-\pi \operatorname{sgn}(\theta)]}\right| \leqslant$ $e^{2 \pi|\nu|},\left|e^{\mathrm{i} z \cos \theta}\right| \leqslant e^{|z|},\left|\gamma^{*}(\nu,-\mathrm{i} z(1-\cos \theta))\right| \leqslant \frac{e^{2|z|}}{|\Gamma(\nu+1)|}$, and $\left|[-\mathrm{i} z(1-\cos \theta)]^{\nu}\right| \leqslant$ $|z|^{|\nu|} e^{\pi|\nu|}(1-\cos \theta)^{\operatorname{Re} \nu}$. Therefore, from (2.17) it follows:

$$
\left|\widetilde{\mathfrak{J}}_{\ell}^{(\nu)}(z)\right| \leqslant \frac{e^{3 \pi|\nu|} e^{3|z|}|z|^{|\nu|}}{\pi|\Gamma(\nu+1)|} \int_{0}^{\pi}(1-\cos \theta)^{\operatorname{Re} \nu} \mathrm{d} \theta,
$$

the integral being convergent for $\operatorname{Re} \nu>-\frac{1}{2}$. Hence, formula (2.16) defines an analytic function of $z$ on $\mathbb{C} \backslash(-\infty, 0]$ (on $\mathbb{C}$ if $\nu \in \mathbb{N}_{0}$ ) for $\operatorname{Re} \nu>-\frac{1}{2}$. 
Finally, in (2.16) we put $\mu=\ell+\nu(\ell=\langle\operatorname{Re} \mu\rangle$ and $\nu=\{\mu\})$ and obtain the following mixed-type representation for the Bessel function of the first kind of any order $\mu \in \mathbb{C}$ :

$$
\begin{aligned}
J_{\mu}(z)= & \frac{\mathrm{i}_{\{\mu\}-\langle\operatorname{Re} \mu\rangle}}{2 \pi} \int_{-\pi}^{\pi} e^{\mathrm{i}\{\mu\}[\theta-\pi \operatorname{sgn}(\theta)]} e^{\mathrm{i} z \cos \theta} P(\{\mu\},-\mathrm{i} z(1-\cos \theta)) e^{\mathrm{i}\langle\operatorname{Re} \mu\rangle \theta} \mathrm{d} \theta \\
& +\frac{\mathrm{i}^{\langle\operatorname{Re} \mu\rangle}}{\sqrt{\pi}}(2 z)^{\{\mu\}} e^{\mathrm{i} z} \sum_{j=\langle\operatorname{Re} \mu\rangle}^{-1} \frac{\Gamma\left(j+\{\mu\}+\frac{1}{2}\right)(-2 \mathrm{i} z)^{j}}{\Gamma(j-\langle\operatorname{Re} \mu\rangle+1) \Gamma(j+\langle\operatorname{Re} \mu\rangle+1+2\{\mu\})} .
\end{aligned}
$$

Rearranging the integral and re-indexing the summation, $j \rightarrow j+\langle\operatorname{Re} \mu\rangle-1$, we finally obtain formula (2.7).

Corollary 2.2. From (2.7) and the formula in (1.6) it is immediate to obtain the mixed-type representation for the modified Bessel function of the first kind:

$$
\begin{aligned}
I_{\mu}(z)= & \frac{1}{\pi} \int_{0}^{\pi} e^{z \cos \theta} P(\{\mu\}, z(1+\cos \theta)) \cos \mu \theta \mathrm{d} \theta \\
& +\frac{1}{\sqrt{\pi}} e^{-z} \sum_{j=1}^{-\langle\operatorname{Re} \mu\rangle} \frac{\Gamma\left(j+\mu-\frac{1}{2}\right)}{\Gamma(j) \Gamma(j+2 \mu)}(2 z)^{j+\mu-1} \quad(\mu \in \mathbb{C} ;-\pi<\arg z \leqslant \pi / 2) .
\end{aligned}
$$

It is worth noting that the first term in (2.7) coincides with the one given in (1.11), which in that case was supposed to hold only for $\operatorname{Re} \mu>-\frac{1}{2}$. The second term in (2.7) is a finite linear combination of inverse powers of $z$ (in the sense that its exponent $j+\operatorname{Re} \mu-1<0)$ and is non-null only when $\operatorname{Re} \mu \leqslant-\frac{1}{2}$.

For later convenience, we write formula (2.7) as

$$
J_{\mu}(z)=B_{\mu}(z)+\chi_{\mu}(z) \quad(\mu \in \mathbb{C})
$$

where the integral term, which is present for all values of $\mu \in \mathbb{C}$, is

$$
B_{\mu}(z) \doteq \frac{\mathrm{i}^{\mu}}{\pi} \int_{0}^{\pi} e^{-\mathrm{i} z \cos \theta} P(\{\mu\},-\mathrm{i} z(1+\cos \theta)) \cos \mu \theta \mathrm{d} \theta \quad(\mu \in \mathbb{C}),
$$

while the corrective term, which is non-null only when $\operatorname{Re} \mu \leqslant-\frac{1}{2}$, is given by:

$$
\chi_{\mu}(z) \doteq\left\{\begin{array}{lll}
\frac{\mathrm{i}^{\mu}}{\sqrt{\pi}} e^{\mathrm{i} z} \sum_{j=1}^{-\langle\operatorname{Re} \mu\rangle} \frac{\Gamma\left(j+\mu-\frac{1}{2}\right)}{\Gamma(j) \Gamma(j+2 \mu)}(-2 \mathrm{i} z)^{j+\mu-1} & \text { if } & \operatorname{Re} \mu \leqslant-\frac{1}{2} \\
0 & \text { if } & \operatorname{Re} \mu>-\frac{1}{2}
\end{array}\right.
$$

As particular cases of $\chi_{\mu}(z)$, we note that when $\mu \equiv m \in \mathbb{Z}^{-} \doteq\{-1,-2, \ldots\}$ is a negative integer, we have $\chi_{m}(z)=0$ in view of the zero brought by the term $\Gamma^{-1}(j+2 \mu)$. Hence, $\chi_{m}(z)=0$ for any $m \in \mathbb{Z}$. If $\mu \equiv m+\frac{1}{2}$ is a negative halfinteger, $m \in \mathbb{Z}^{-}$, formula (2.24) should be understood in the limiting form in view of the ratio of the two singular terms: $\Gamma(j+m) / \Gamma(j+2 m+1)$. Recalling that

$$
\lim _{\substack{\mu \rightarrow m+\frac{1}{2} \\ m \in \mathbb{Z}^{-}}} \frac{\Gamma\left(j+\mu-\frac{1}{2}\right)}{\Gamma(j+2 \mu)}=2(-1)^{m+1} \frac{\Gamma(-j-2 m)}{\Gamma(-j-m+1)},
$$


we have for $m \in \mathbb{Z}$ :

$(2.26)$

$$
\chi_{m+\frac{1}{2}}(z) \doteq \begin{cases}\frac{2}{\sqrt{\pi}}(-\mathrm{i})^{m+\frac{3}{2}} e^{\mathrm{i} z} \sum_{j=1}^{-m} \frac{\Gamma(j-m-1)}{\Gamma(j) \Gamma(-j-m+1)}(-2 \mathrm{i} z)^{\frac{1}{2}-j} & \text { if } \quad m \leqslant-1 \\ 0 & \text { if } \quad m \geqslant 0 .\end{cases}
$$

It is also useful for what follows to analyze the limiting forms of $\chi_{\mu}(z)$ for both $z \rightarrow 0, \infty$. From (2.24) we see that near the origin $\chi_{\mu}(z)$ diverges as

$$
\chi_{\mu}(z) \underset{z \rightarrow 0}{\sim} \frac{1}{\Gamma(1+\mu)}\left(\frac{z}{2}\right)^{\mu} \quad\left(\operatorname{Re} \mu \leqslant-\frac{1}{2}\right) .
$$

For large values of $|z|, \chi_{\mu}(z)$ is easily seen from (2.24) to vanish as:

$$
\chi_{\mu}(z) \underset{z \rightarrow \infty}{\sim} \frac{\mathrm{i}^{1+\langle\operatorname{Re} \mu\rangle}}{\sqrt{\pi}} \frac{\Gamma\left(\{\mu\}-\frac{1}{2}\right)}{\Gamma(-\langle\operatorname{Re} \mu\rangle) \Gamma(\mu+\{\mu\})}(2 z)^{\{\mu\}-1} e^{\mathrm{i} z} \quad\left(\operatorname{Re} \mu \leqslant-\frac{1}{2}\right) .
$$

In order to analyze the behavior of $B_{\mu}(z)$ for $z \rightarrow 0$, we first recall that the regularized incomplete gamma function can be written as: $P(\mu, w)=w^{\mu} \gamma^{*}(\mu, w)$ $($ see (2.18) $)$. Now, in a neighborhood of the origin $\gamma^{*}(\mu, w)$ is bounded by $\gamma^{*}(\mu, 0)=$ $1 / \Gamma(\mu+1)$. Then, we can write:

$$
P(\mu, w) \underset{w \rightarrow 0}{\sim} \begin{cases}\frac{w^{\mu}}{\Gamma(\mu+1)} & \text { if } \quad \mu \neq-1,-2, \ldots, \\ 1 & \text { if } \quad \mu=-1,-2, \ldots\end{cases}
$$

Inserting in formula (2.23) the approximation (2.29) along with the $N$ th order Taylor approximation: $e^{-\mathrm{i} z \cos \theta} \simeq \sum_{j=0}^{N}(-\mathrm{i} z \cos \theta)^{j} / j$ !, we obtain:

$B_{\mu}(z) \underset{z \rightarrow 0}{\sim} \frac{\mathrm{i}^{\mu}}{\pi} \frac{(-\mathrm{i} z)^{\{\mu\}}}{\Gamma(1+\{\mu\})} \sum_{j=0}^{N} \frac{(-\mathrm{i} z)^{j}}{j !} \int_{0}^{\pi}(\cos \theta)^{j}(1+\cos \theta)^{\{\mu\}} \cos (\langle\operatorname{Re} \mu\rangle+\{\mu\}) \theta \mathrm{d} \theta$,

where we have written $\mu$ in terms of its integral and fractional parts. Now, the integrals in (2.30) are null for $j<\langle\operatorname{Re} \mu\rangle$ (since they are proportional to $\Gamma^{-1}(j+$ $1-\langle\operatorname{Re} \mu\rangle))$. Then, it must be $N \geqslant\langle\operatorname{Re} \mu\rangle$ and, as first approximation (i.e., putting $N=\langle\operatorname{Re} \mu\rangle)$ it yields:

$$
B_{\mu}(z) \underset{z \rightarrow 0}{\sim} \frac{c_{\mu}}{\pi \Gamma(1+\langle\operatorname{Re} \mu\rangle) \Gamma(1+\{\mu\})} z^{\mu},
$$

where

$$
c_{\mu}=\int_{0}^{\pi}(\cos \theta)^{\langle\operatorname{Re} \mu\rangle}(1+\cos \theta)^{\{\mu\}} \cos \mu \theta \mathrm{d} \theta \quad(\langle\operatorname{Re} \mu\rangle \geqslant 0) .
$$

The computation of this latter integral can be done by using formula (2.13) and the suitable trigonometric power formula for the term $(\cos \theta)^{\langle\operatorname{Re} \mu\rangle}$. We obtain: $c_{\mu}=\pi / 2^{\mu}$. Then, from (2.22) and using (2.27) we have:

$$
J_{\mu}(z) \underset{z \rightarrow 0}{\sim}\left\{\begin{array}{lll}
\frac{1}{\Gamma(1+\langle\operatorname{Re} \mu\rangle) \Gamma(1+\{\mu\})}\left(\frac{z}{2}\right)^{\mu} & \text { if } \quad \operatorname{Re} \mu>-\frac{1}{2}, \\
\frac{1}{\Gamma(1+\mu)}\left(\frac{z}{2}\right)^{\mu} & \text { if } \quad \operatorname{Re} \mu \leqslant-\frac{1}{2} \quad(\mu \neq-1,-2, \ldots) .
\end{array}\right.
$$


Numerical tests show that, for $\operatorname{Re} \mu>-\frac{1}{2}$, the classical limiting approximation of $J_{\mu}(z)$, as $z \rightarrow 0$, performs better than the one given in (2.33).

2.1. Representation of $\boldsymbol{J}_{\boldsymbol{m}}(\boldsymbol{z})$ with $\boldsymbol{m} \in \mathbb{Z}$. Let $\mu=m \in \mathbb{Z}$. Equation (2.22) gives no new information. In fact, we have $\chi_{m}(z)=0$ for all $m \in \mathbb{Z}$. For what concerns $B_{m}(z)$ in (2.23), it yields the well-known Jacobi-Anger integral representation of $J_{m}(z)$, i.e.:

$$
B_{m}(z)=\frac{\mathrm{i}^{m}}{\pi} \int_{0}^{\pi} e^{-\mathrm{i} z \cos \theta} \cos (m \theta) \mathrm{d} \theta \equiv J_{m}(z) .
$$

2.2. Representation of the spherical Bessel function of the first kind and Dawson's integral. Let $\mu=m+\frac{1}{2}, m \in \mathbb{Z}$. The integral term (2.23) simply reads:

$$
B_{m+\frac{1}{2}}(z)=\frac{\mathrm{i}^{m+\frac{1}{2}}}{\pi} \int_{0}^{\pi} e^{-\mathrm{i} z \cos \theta} P\left(\frac{1}{2},-\mathrm{i} z(1+\cos \theta)\right) \cos \left(m+\frac{1}{2}\right) \theta \mathrm{d} \theta \quad(m \in \mathbb{Z}) .
$$

This latter formula can be written differently recalling that $P\left(\frac{1}{2}, w^{2}\right)=\operatorname{erf}(w)$, where $\operatorname{erf}(w)$ denotes the error function [7, Eq. 7.2.1]. Then, we have:

$$
B_{m+\frac{1}{2}}(z)=\frac{\mathrm{i}^{m+\frac{1}{2}}}{\pi} \int_{0}^{\pi} e^{-\mathrm{i} z \cos \theta} \operatorname{erf}(\sqrt{-2 \mathrm{i} z} \cos (\theta / 2)) \cos \left(m+\frac{1}{2}\right) \theta \mathrm{d} \theta .
$$

Notice that $B_{m+\frac{1}{2}}(z)$ is related to the $m$ th Fourier coefficient $\tilde{g}_{m}(z)$ of the $2 \pi$ periodic function $g_{z}(\theta)=\frac{1}{2 \pi} \exp (-\mathrm{i}(z \cos \theta-\theta / 2) \operatorname{erf}(\sqrt{-2 \mathrm{i} z} \cos (\theta / 2))$. Precisely, we have: $(-\mathrm{i})^{m+\frac{1}{2}} B_{m+\frac{1}{2}}(z)=\tilde{g}_{m}(z)$. Therefore, we can now invert the Fourier representation (see (2.1)) and, using the symmetry $\tilde{g}_{m}(z)=\tilde{g}_{-m-1}(z)$, setting $w=\sqrt{-2 \mathrm{i} z}$ and recalling that $B_{m+\frac{1}{2}}(z)=J_{m+\frac{1}{2}}(z)$ for $m \geqslant 0$ (see (2.22) and (2.26)), we have thus the following representation for the error function 1 :

$$
\operatorname{erf}(w \cos (\theta / 2))=2 e^{-\frac{1}{2} w^{2} \cos \theta} \sum_{m=0}^{\infty} I_{m+\frac{1}{2}}\left(w^{2} / 2\right) \cos \left(m+\frac{1}{2}\right) \theta
$$

where $I_{\mu}(z)$ denotes the modified Bessel function of the first kind. Expression (2.36) can be written also in terms of Dawson's integral $F(z)$ [7, Eq. 7.2.5]:

$$
F(z) \doteq e^{-z^{2}} \int_{0}^{z} e^{t^{2}} \mathrm{~d} t
$$

We have:

$$
B_{m+\frac{1}{2}}(z)=\frac{2 \mathrm{i}^{m+3 / 2} e^{\mathrm{i} z}}{\pi \sqrt{\pi}} \int_{0}^{\pi} F(-\mathrm{i} \sqrt{-2 \mathrm{i} z} \cos (\theta / 2)) \cos \left(m+\frac{1}{2}\right) \theta \mathrm{d} \theta \quad(m \in \mathbb{Z}) .
$$

\footnotetext{
${ }^{1}$ From [2.37), representations [6, Eq. (9.4.20) p. 57; Eq. (9.4.21) p. 58] and [7 Eq. 7.6.8] easily follow.
} 
Finally, from (2.26) and (2.39), and recalling the definition [7, Eq. 10.47.3]: $j_{m}(z)=$ $\sqrt{\frac{1}{2} \pi / z} J_{m+\frac{1}{2}}(z)$, we have the following representation for the spherical Bessel function of the first kind $j_{m}(z)$ :

$$
\begin{aligned}
j_{m}(z)= & \sqrt{2} \mathrm{i}^{m+\frac{3}{2}} \frac{e^{\mathrm{i} z}}{\sqrt{z}}\left[\frac{1}{\pi} \int_{0}^{\pi} F(-\mathrm{i} \sqrt{-2 \mathrm{i} z} \cos (\theta / 2)) \cos \left(m+\frac{1}{2}\right) \theta \mathrm{d} \theta\right. \\
& \left.+\mathrm{i}(-1)^{m} \sum_{j=1}^{-m} \frac{\Gamma(j-m-1)}{\Gamma(j) \Gamma(-j-m+1)}(-2 \mathrm{i} z)^{\frac{1}{2}-j}\right] \quad(m \in \mathbb{Z}) .
\end{aligned}
$$

As a by-product, assuming $m \geqslant 0$ in (2.40) we readily get the coefficient of the cosine transform of the function $F(w \cos (\theta / 2))$ :

$$
\frac{1}{\pi} \int_{0}^{\pi} F(w \cos (\theta / 2)) \cos \left(m+\frac{1}{2}\right) \theta \mathrm{d} \theta=\frac{w e^{-w^{2} / 2}}{2 \mathrm{i}^{m}} j_{m}\left(-\mathrm{i} w^{2} / 2\right) \quad(m \geqslant 0),
$$

which, inverting the cosine transform, yields the following representation of Dawson's integral:

$$
F(w \cos \theta)=w e^{-w^{2} / 2} \sum_{m=0}^{\infty}(-\mathrm{i})^{m} j_{m}\left(-\mathrm{i} w^{2} / 2\right) \cos (2 m+1) \theta .
$$

A duplication formula for the Dawson integral can be obtained from (2.42). First we put $\theta=0$ in (2.42) to get

$$
F(w)=w e^{-w^{2} / 2} \sum_{m=0}^{\infty}(-\mathrm{i})^{m} j_{m}\left(-\mathrm{i} w^{2} / 2\right) .
$$

Then, expression (2.42) with $\theta=\pi / 3$ along with (2.43) yields the duplication formula:

$$
F(2 w)=2 F(w)+6 w e^{-2 w^{2}} \sum_{m=0}^{\infty}(-1)^{m}\left[j_{6 m+4}\left(-2 \mathrm{i} w^{2}\right)-\mathrm{i} j_{6 m+1}\left(-2 \mathrm{i} w^{2}\right)\right]
$$

2.3. Representation of the derivative $\partial J_{\mu}(z) / \partial z$. The representation for the derivatives of the Bessel function $J_{\mu}(z)$ is easily obtained from formula [7, Eq. 10.6.1]:

$$
J_{\mu}^{(1)}(z) \equiv \frac{\partial J_{\mu}}{\partial z}=\frac{1}{2}\left[J_{\mu-1}(z)-J_{\mu+1}(z)\right] .
$$

By using (2.22) and exploiting the fact that the regularized incomplete gamma function in (2.23) depends on the fractional part of $\mu$, we can write:

$$
\begin{aligned}
J_{\mu}^{(1)}(z)= & \frac{\mathrm{i}^{\mu-1}}{\pi} \int_{0}^{\pi} e^{-\mathrm{i} z \cos \theta} P(\{\mu\},-\mathrm{i} z(1+\cos \theta)) \cos \theta \cos \mu \theta \mathrm{d} \theta \\
& +\frac{1}{2}\left[\chi_{\mu-1}(z)-\chi_{\mu+1}(z)\right] .
\end{aligned}
$$

The generalization to any integer order $n \geqslant 0$ is immediate by iterating formula (2.45) and taking into account the expression of the $n$th order central differences 
[1. p. 877]. We have:

$$
\begin{aligned}
J_{\mu}^{(n)}(z)= & \frac{\mathrm{i}^{\mu-n}}{\pi} \int_{0}^{\pi} e^{-\mathrm{i} z \cos \theta} P(\{\mu\},-\mathrm{i} z(1+\cos \theta))(\cos \theta)^{n} \cos \mu \theta \mathrm{d} \theta \\
& +\frac{1}{2^{n}} \sum_{j=0}^{n}(-1)^{j}\left(\begin{array}{c}
n \\
j
\end{array}\right) \chi_{\mu-n+2 j}(z) \quad(n \geqslant 0) .
\end{aligned}
$$

It is worth noting that, in view of definition (2.24) of $\chi_{\mu}(z)$, the sum term in (2.47) is null for $\langle\operatorname{Re} \mu\rangle \geqslant n$.

\section{Representation of Neumann's function $Y_{\mu}(z)$}

Since formula (2.7) holds true for any complex value of $\mu$, a similar mixed-type representation can be written also for the Bessel function of the second type $Y_{\mu}(z)$ by means of formula (1.3). Therefore, from (2.22) and recalling that the term $\chi_{\mu}(z)$ is zero if $\operatorname{Re} \mu>-\frac{1}{2}$ (see (2.24)), we have, for any $\mu \in \mathbb{C}$, the following representation of the Bessel function of the second kind which, even in this case, has the structure of an integral term plus a finite sum:

$$
Y_{\mu}(z)=y_{\mu}(z)+S(\mu) \frac{\chi_{[-\mu \operatorname{sgn}(\operatorname{Re} \mu)]}(z)}{\sin \mu \pi} \quad(\mu \in \mathbb{C}),
$$

where the integral term is given by:

$$
\begin{aligned}
& y_{\mu}(z) \doteq \frac{B_{\mu}(z) \cos \mu \pi-B_{-\mu}(z)}{\sin \mu \pi}=\frac{\mathrm{i}^{\mu}}{\pi \sin \mu \pi} \int_{0}^{\pi} e^{-\mathrm{i} z \cos \theta} \\
& \cdot\left[\cos \mu \pi P(\{\mu\},-\mathrm{i} z(1+\cos \theta))-e^{-\mathrm{i} \mu \pi} P(-\{\mu\},-\mathrm{i} z(1+\cos \theta))\right] \cos \mu \theta \mathrm{d} \theta, \\
& S(\mu) \doteq\left\{\begin{array}{lll}
\cos \mu \pi & \text { if } \quad & \operatorname{Re} \mu<0, \\
-1 & \text { if } \quad & \operatorname{Re} \mu \geqslant 0,
\end{array}\right.
\end{aligned}
$$

and $\chi_{\nu}(z)$ is given in (2.24). Note that $\chi_{[-\mu \operatorname{sgn}(\operatorname{Re} \mu)]}(z)=0$ for $|\operatorname{Re} \mu|<\frac{1}{2}$.

3.1. Representation of $\boldsymbol{Y}_{\boldsymbol{m}}(\boldsymbol{z})$ with $\boldsymbol{m} \in \mathbb{Z}$. The Bessel function of the second kind is particularly useful when $\mu \equiv m$ is an integer since, in this case, we have $J_{-m}(z)=(-1)^{m} J_{m}(z)$ and $Y_{m}(z)$ represents the second independent solution to the Bessel equation (1.1). The mixed-type representation for $Y_{m}(z)$ can be obtained evaluating the limit of (3.1) for $\mu \rightarrow m$. Recalling that $\mu=\langle\operatorname{Re} \mu\rangle+\{\mu\}$, this limit can be written by setting $\langle\operatorname{Re} \mu\rangle=m$ and taking the limit $\{\mu\} \rightarrow 0$. Let us consider the case $\operatorname{Re} \mu \geqslant 1$, the analysis when $\operatorname{Re} \mu \leqslant-1$ being strictly similar. The case $\mu \rightarrow 0$ is studied separately. We have from (3.1):

$$
Y_{m}(z)=\lim _{\substack{\langle\operatorname{Re} \mu\rangle=m \geqslant 1 \\\{\mu\} \rightarrow 0}} Y_{\mu}(z)=\lim _{\substack{\langle\operatorname{Re} \mu=m \geqslant 1 \\\{\mu\} \rightarrow 0}} y_{\mu}(z)-\lim _{\substack{\langle\operatorname{Re} \mu\rangle=m \geqslant 1 \\\{\mu\} \rightarrow 0}} \frac{\chi_{-\mu}(z)}{\sin \mu \pi},
$$

with the latter two limits existing finite. For what concerns the first limit on the r.h.s. of (3.4), we recall that $P(0, w)=1$ and, moreover,

$$
\lim _{a \rightarrow 0} \frac{P(a, w)-P(-a, w)}{\sin \pi a}=-\frac{2}{\pi} \Gamma(0, w),
$$


where $\Gamma(\nu, w)$ denotes the upper incomplete gamma function [7, Eq. 8.2.2]. Therefore, from (3.2) we obtain:

$$
\begin{aligned}
& y_{m}(z)=\lim _{\substack{\langle\operatorname{Re} \mu\rangle=m \geqslant 1 \\
\{\mu\} \rightarrow 0}} y_{\mu}(z) \\
& =\frac{\mathrm{i}^{m}}{\pi} \int_{0}^{\pi} e^{-\mathrm{i} z \cos \theta} \cos m \theta \lim _{\{\mu\} \rightarrow 0} \frac{P(\{\mu\},-\mathrm{i} z(1+\cos \theta))-P(-\{\mu\},-\mathrm{i} z(1+\cos \theta))}{\sin \{\mu\} \pi} \mathrm{d} \theta \\
& \quad+\frac{\mathrm{i}^{m+1}}{\pi} \int_{0}^{\pi} e^{-\mathrm{i} z \cos \theta} \cos m \theta \lim _{\{\mu\} \rightarrow 0} P(-\{\mu\},-\mathrm{i} z(1+\cos \theta)) \mathrm{d} \theta \\
& =\frac{2 \mathrm{i}^{m}}{\pi^{2}} \int_{0}^{\pi} e^{-\mathrm{i} z \cos \theta} \cos m \theta\left[\mathrm{i} \frac{\pi}{2}-\Gamma(0,-\mathrm{i} z(1+\cos \theta))\right] \mathrm{d} \theta \quad(m \geqslant 1) .
\end{aligned}
$$

Recalling representation (2.34) of $J_{m}(z)$, formula (3.6) can also be written as:

$$
y_{m}(z)=-\frac{2 \mathrm{i}^{m}}{\pi^{2}} \int_{0}^{\pi} e^{-\mathrm{i} z \cos \theta} \Gamma(0,-\mathrm{i} z(1+\cos \theta)) \cos m \theta \mathrm{d} \theta+\mathrm{i} J_{m}(z) .
$$

Concerning the second limit on the r.h.s of (3.4), we have from (2.24):

$$
\begin{aligned}
& \lim _{\substack{\langle\operatorname{Re} \mu\rangle=m \geqslant 1 \\
\{\mu\} \rightarrow 0}} \frac{\chi_{-\mu}(z)}{\sin \mu \pi} \\
& =\frac{\mathrm{i}^{-m}}{\sqrt{\pi}}(-2 \mathrm{i} z)^{-m-1} e^{\mathrm{i} z} \sum_{j=1}^{m}(-2 \mathrm{i} z)^{j} \frac{\Gamma\left(j-m-\frac{1}{2}\right)}{\Gamma(j)} \lim _{\substack{\langle\operatorname{Re} \mu\rangle=m \geqslant 1 \\
\{\mu\} \rightarrow 0}} \frac{1}{\Gamma(j-2 \mu) \sin \mu \pi} \\
& =\frac{2 e^{\mathrm{i} z}}{\mathrm{i}^{m} \pi \sqrt{\pi}} \sum_{j=1}^{m} \frac{\Gamma\left(\frac{1}{2}-j\right) \Gamma(m+j)}{\Gamma(m-j+1)}(2 \mathrm{i} z)^{-j} \quad(m \geqslant 1),
\end{aligned}
$$

where we used

$$
\lim _{\substack{\langle\operatorname{Re} \mu\rangle=m \geqslant 1 \\\{\mu\} \rightarrow 0}} \Gamma(j-2 \mu) \sin \mu \pi=(-1)^{m+j+1} \frac{\pi}{2 \Gamma(2 m+1-j)},
$$

and re-inxeded the sum by setting: $j \rightarrow-j+m+1$. Finally, plugging formulae (3.6) and (3.8) into (3.4) yields the following representation for the Bessel function of the second kind and integer order $m \geqslant 1$ :

$$
\begin{aligned}
Y_{m}(z)= & \frac{2 \mathrm{i}^{m}}{\pi}\left\{\frac{1}{\pi} \int_{0}^{\pi} e^{-\mathrm{i} z \cos \theta}\left[\mathrm{i} \frac{\pi}{2}-\Gamma(0,-\mathrm{i} z(1+\cos \theta))\right] \cos m \theta \mathrm{d} \theta\right. \\
& \left.-\frac{(-1)^{m}}{\sqrt{\pi}} e^{\mathrm{i} z} \sum_{j=1}^{m} \frac{\Gamma\left(\frac{1}{2}-j\right) \Gamma(j+m)}{\Gamma(m-j+1)}(2 \mathrm{i} z)^{-j}\right\} \quad(m \geqslant 1) .
\end{aligned}
$$

The case $m \leqslant-1$ can be treated analogously, the only difference is that in the sum term $m \rightarrow-m$, the integral term remaining unchanged. In view of (3.1), when $m=0$ only the integral part is non-null. Thus, we finally obtain the following representation for $m \in \mathbb{Z}$ :

$$
Y_{m}(z)=-\frac{2 \mathrm{i}^{m}}{\pi}\left\{\frac{1}{\pi} \int_{0}^{\pi} e^{-\mathrm{i} z \cos \theta}\left[\Gamma(0,-\mathrm{i} z(1+\cos \theta))-\mathrm{i} \frac{\pi}{2}\right] \cos m \theta \mathrm{d} \theta+\sigma_{m}(z)\right\},
$$


where

$$
\sigma_{m}(z)=\frac{(-1)^{m}}{\sqrt{\pi}} e^{\mathrm{i} z} \sum_{j=1}^{|m|} \frac{\Gamma\left(\frac{1}{2}-j\right) \Gamma(j+|m|)}{\Gamma(|m|-j+1)}(2 \mathrm{i} z)^{-j},
$$

the sum being understood to be zero when $m=0$. Explicitly, this latter case reads:

$$
Y_{0}(z)=\frac{2}{\pi^{2}} \int_{0}^{\pi} e^{-\mathrm{i} z \cos \theta}\left[\mathrm{i} \frac{\pi}{2}-\Gamma(0,-\mathrm{i} z(1+\cos \theta))\right] \mathrm{d} \theta .
$$

Formula (3.7), along with formulae (3.4) and (3.8), readily yields also a new representation for the Hankel function of the first type of integral order $H_{m}^{(1)}(z), m \in \mathbb{Z}$ (see formula (1.5) ):

$H_{m}^{(1)}(z)=\frac{2 \mathrm{i}^{m-1}}{\pi}\left[\frac{1}{\pi} \int_{0}^{\pi} e^{-\mathrm{i} z \cos \theta} \Gamma(0,-\mathrm{i} z(1+\cos \theta)) \cos m \theta \mathrm{d} \theta+\sigma_{m}(z)\right],(m \in \mathbb{Z})$.

The general case of Hankel functions of complex order $\mu$ will be given in Section 4

3.2. Representation of the spherical Bessel function of the second kind. We start from formula (3.1) with $\mu=m+\frac{1}{2}, m \in \mathbb{Z}$. From (3.2) we see that $y_{m+\frac{1}{2}}(z)=(-1)^{m+1} B_{-m-\frac{1}{2}}(z)$ and, using (2.23), we obtain (see also (2.39) ):

$$
y_{m+\frac{1}{2}}(z)=-\frac{2 \mathrm{i}^{m+\frac{1}{2}}}{\pi \sqrt{\pi}} e^{\mathrm{i} z} \int_{0}^{\pi} F(-\mathrm{i} \sqrt{-2 \mathrm{i} z} \cos (\theta / 2)) \cos \left(m+\frac{1}{2}\right) \theta \mathrm{d} \theta .
$$

For what regards the sum term in (3.1), we see from (3.3) that it does not contribute for $m \leqslant-1$ since in this case $S\left(m+\frac{1}{2}\right)=0$. Then, we limit ourselves to consider the sum term for $m \geqslant 0$. From (2.24) and recalling that for $\mu=-m-\frac{1}{2}$ its integral and fractional parts are respectively $\langle\operatorname{Re} \mu\rangle=-m-1$ and $\{\mu\}=\frac{1}{2}$, we obtain:

$$
\begin{aligned}
\chi_{-m-\frac{1}{2}}(z) & =\frac{\mathrm{i}^{-m-\frac{1}{2}}}{\sqrt{\pi}} e^{\mathrm{i} z} \sum_{j=1}^{m+1} \frac{(-2 \mathrm{i} z)^{j-m-\frac{3}{2}}}{\Gamma(j)} \lim _{\substack{\nu \rightarrow m \\
m=0,1,2, \ldots}} \frac{\Gamma(j-\nu-1)}{\Gamma(j-2 \nu-1)} \\
& =\frac{2 \mathrm{i}^{m-\frac{1}{2}}}{\sqrt{\pi}} e^{\mathrm{i} z} \sum_{j=1}^{m+1} \frac{\Gamma(2 m-j+2)}{\Gamma(j) \Gamma(m-j+2)}(-2 \mathrm{i} z)^{j-m-\frac{3}{2}} \quad(m \geqslant 0),
\end{aligned}
$$

where we used the limit

$$
\lim _{\substack{\nu \rightarrow m \\ m=0,1,2, \ldots}} \frac{\Gamma(j-\nu-1)}{\Gamma(j-2 \nu-1)}=2(-1)^{m} \frac{(2 m-j+1) !}{(m-j+1) !} \quad(1 \leqslant j \leqslant m+1) .
$$

Now, recalling that the spherical Bessel function of the second kind is given by: $y_{m}(z)=\sqrt{\pi /(2 z)} Y_{m+\frac{1}{2}}(z)$, we can finally write:

$$
\begin{aligned}
y_{m}(z)= & \sqrt{\frac{2 \mathrm{i}}{z}} e^{\mathrm{i} z} \mathrm{i}^{m}\left[-\frac{1}{\pi} \int_{0}^{\pi} F(-\mathrm{i} \sqrt{-2 \mathrm{i} z} \cos (\theta / 2)) \cos \left(m+\frac{1}{2}\right) \theta \mathrm{d} \theta\right. \\
& \left.+\mathrm{i}(-1)^{m} \sum_{j=0}^{m} \frac{(2 m-j) !}{j !(m-j) !}(-2 \mathrm{i} z)^{j-m-\frac{1}{2}}\right] \quad(m \in \mathbb{Z}) .
\end{aligned}
$$


3.3. Limiting form of $\boldsymbol{Y}_{\boldsymbol{\mu}}(\boldsymbol{z})$ near $\boldsymbol{z}=\mathbf{0}$. Referring to (3.1), let us first consider the behavior near the origin $z=0$ of the sum term in formula (3.1). In view of (2.27), we see that this term diverges near $z=0$ as $O\left(z^{-|\operatorname{Re} \mu|}\right)$. Precisely, if $\mu$ is not integer, we have:

$$
\chi_{[-\mu \operatorname{sgn}(\operatorname{Re} \mu)]}(z) \underset{z \rightarrow 0}{\sim} \frac{1}{\Gamma(1-\mu \operatorname{sgn}(\operatorname{Re} \mu))}\left(\frac{2}{z}\right)^{\mu \operatorname{sgn}(\operatorname{Re} \mu)} \quad(\mu \in \mathbb{C} \backslash \mathbb{Z}) .
$$

For what concerns the integral term $y_{\mu}(z)$ in (3.1), we first recall that the regularized incomplete gamma function can be written as: $P(\mu, w)=w^{\mu} \gamma^{*}(\mu, w)$ (see (2.18)). Since in (3.2) we have: $-\frac{1}{2}<\operatorname{Re}\{\mu\}<\frac{1}{2}$, for the moment we limit ourselves to consider the case $\mu \neq 0$. The study of the case $\mu=0$ will be considered later. From (2.29) we have:

$$
P(\{\mu\},-\mathrm{i} z(1+\cos \theta)) \underset{z \rightarrow 0}{\sim}\left\{\begin{array}{lll}
0 & \text { if } & 0<\operatorname{Re}\{\mu\}<\frac{1}{2}, \\
\frac{[-\mathrm{i} z(1+\cos \theta)]^{\{\mu\}}}{\Gamma(1+\{\mu\})} & \text { if } \quad-\frac{1}{2}<\operatorname{Re}\{\mu\}<0 .
\end{array}\right.
$$

Analogously,

$$
P(-\{\mu\},-\mathrm{i} z(1+\cos \theta)) \underset{z \rightarrow 0}{\sim}\left\{\begin{array}{lll}
\frac{[-\mathrm{i} z(1+\cos \theta)]^{-\{\mu\}}}{\Gamma(1-\{\mu\})} & \text { if } \quad 0<\operatorname{Re}\{\mu\}<\frac{1}{2}, \\
0 & \text { if } \quad-\frac{1}{2}<\operatorname{Re}\{\mu\}<0,
\end{array}\right.
$$

Then, we recall the following integral:

$$
\int_{0}^{\pi} \frac{\cos \mu \theta}{(1+\cos \theta)^{\{\mu\}}} \mathrm{d} \theta=\frac{2^{\{\mu\}} \pi \Gamma(1-2\{\mu\})}{\Gamma(1-\mu-\{\mu\}) \Gamma(1+\mu-\{\mu\})}, \quad\left(\operatorname{Re}\{\mu\}<\frac{1}{2}\right) .
$$

Assume for the moment $0<\operatorname{Re}\{\mu\}<\frac{1}{2}$; the case $-\frac{1}{2}<\operatorname{Re}\{\mu\}<0$ is strictly analogous. Using (3.21), (3.22), and noting that $e^{-\mathrm{i} z \cos \theta} \rightarrow 1$ as $z \rightarrow 0$, we have from (3.2):

$$
\begin{aligned}
& y_{\mu}(z) \doteq \frac{\mathrm{i}^{\mu}}{\pi \sin \mu \pi} \int_{0}^{\pi} e^{-\mathrm{i} z \cos \theta} \cos \mu \theta \\
& \cdot\left[P(\{\mu\},-\mathrm{i} z(1+\cos \theta)) \cos \mu \pi-e^{-\mathrm{i} \mu \pi} P(-\{\mu\},-\mathrm{i} z(1+\cos \theta))\right] \mathrm{d} \theta \\
& \underset{z \rightarrow 0}{\sim}-\frac{\mathrm{i}^{-\mu}}{\pi \sin \mu \pi} \frac{(-\mathrm{i} z)^{-\{\mu\}}}{\Gamma(1-\{\mu\})} \int_{0}^{\pi} \frac{\cos \mu \theta}{(1+\cos \theta)^{\{\mu\}}} \mathrm{d} \theta \\
&=-\frac{\mathrm{i}^{-\mu}}{\sqrt{\pi} \sin \mu \pi} \frac{\Gamma\left(\frac{1}{2}-\{\mu\}\right)}{\Gamma(1-\mu-\{\mu\}) \Gamma(1+\mu-\{\mu\})}\left(\frac{\mathrm{i}}{2 z}\right)^{\{\mu\}}\left(0<\operatorname{Re}\{\mu\}<\frac{1}{2}\right) .
\end{aligned}
$$

The case $-\frac{1}{2}<\operatorname{Re}\{\mu\}<0$ is treated analogously. Summarizing, the approximation to (3.2) in the vicinity of $z=0$ can be written for $|\operatorname{Re} \mu| \geqslant \frac{1}{2}$ :

$$
y_{\mu}(z) \underset{z \rightarrow 0}{\sim}\left\{\begin{array}{l}
\frac{\mathrm{i}^{\mu} \cos \mu \pi}{\sqrt{\pi} \sin \mu \pi} \frac{\Gamma\left(\frac{1}{2}+\{\mu\}\right)}{\Gamma(1-\mu+\{\mu\}) \Gamma(1+\mu+\{\mu\})}\left(\frac{2 z}{\mathrm{i}}\right)^{\{\mu\}},-\frac{1}{2}<\operatorname{Re}\{\mu\}<0, \\
\frac{-\mathrm{i}^{-\mu}}{\sqrt{\pi} \sin \mu \pi} \frac{\Gamma\left(\frac{1}{2}-\{\mu\}\right)}{\Gamma(1-\mu-\{\mu\}) \Gamma(1+\mu-\{\mu\})}\left(\frac{\mathrm{i}}{2 z}\right)^{\{\mu\}}, \quad 0<\operatorname{Re}\{\mu\}<\frac{1}{2} .
\end{array}\right.
$$


Therefore, in the vicinity of $z=0$ we have: $y_{\mu}(z)=O\left(z^{-|\operatorname{Re}\{\mu\}|}\right)$, which is negligeable with respect to the term $\Sigma_{[-\mu \operatorname{sgn}(\operatorname{Re} \mu)]}(z)$ of formula $(3.19)$. When $|\operatorname{Re} \mu|<\frac{1}{2}$ the latter sum term is null (see (3.3) ) and the behavior near $z=0$ is governed only by the integral term (3.24) with $\mu=\{\mu\}$. Then, the approximation for $|\operatorname{Re} \mu|<\frac{1}{2}$ reads:

$$
y_{\mu}(z) \underset{z \rightarrow 0}{\sim} \begin{cases}\frac{\cos \mu \pi}{\sin \mu \pi} \frac{1}{\Gamma(1+\mu)}\left(\frac{z}{2}\right)^{\mu} & \text { if } \quad-\frac{1}{2}<\operatorname{Re} \mu<0, \\ -\frac{1}{\sin \mu \pi} \frac{1}{\Gamma(1-\mu)}\left(\frac{z}{2}\right)^{-\mu} & \text { if } \quad 0<\operatorname{Re} \mu<\frac{1}{2} .\end{cases}
$$

Written in more compact form, we have:

$Y_{\mu}(z) \underset{z \rightarrow 0}{\sim} \frac{S(\mu)}{\sin \mu \pi} \frac{1}{\Gamma(1-\mu \operatorname{sgn}(\operatorname{Re} \mu))}\left(\frac{2}{z}\right)^{\mu \operatorname{sgn}(\operatorname{Re} \mu)} \quad\left(z, \mu \in \mathbb{C} ;|\operatorname{Re} \mu|<\frac{1}{2}, \mu \neq 0\right)$,

where $S(\mu)$ is given in (3.3). Note that formula (3.26) has the same structure as formula (3.19) which holds for any complex (not integer) value of $\mu$. Therefore, from (3.1), (3.19) and (3.26) we can extend the validity of (3.26) to the entire complex $\mu$-plane (but integers) and write:

$$
Y_{\mu}(z) \underset{z \rightarrow 0}{\sim} \frac{S(\mu)}{\sin \mu \pi} \frac{1}{\Gamma(1-\mu \operatorname{sgn}(\operatorname{Re} \mu))}\left(\frac{2}{z}\right)^{\mu \operatorname{sgn}(\operatorname{Re} \mu)} \quad(z, \mu \in \mathbb{C}, \mu \notin \mathbb{Z}) .
$$

Finally, we use the formula $\Gamma(z) \Gamma(1-z)=\pi / \sin \pi z$ to obtain the final form (see [7. Eqs. 10.7.4, 10.7.5]:

$$
Y_{\mu}(z) \underset{z \rightarrow 0}{\sim} \frac{\operatorname{sgn}(\operatorname{Re} \mu) S(\mu)}{\pi} \Gamma(\mu \operatorname{sgn}(\operatorname{Re} \mu))\left(\frac{2}{z}\right)^{\mu \operatorname{sgn}(\operatorname{Re} \mu)} \quad(z, \mu \in \mathbb{C}, \mu \notin \mathbb{Z}) .
$$

The limiting behavior of $Y_{\mu}(z)$ when $\mu \equiv m \in \mathbb{Z}$ is easily seen by considering formula (3.11). We first observe that, for $m \neq 0$, the integral term in (3.11) is finite when $z \rightarrow 0$, precisely:

$$
\frac{2 \mathrm{i}^{m}}{\pi^{2}} \int_{0}^{\pi} e^{-\mathrm{i} z \cos \theta}\left[\mathrm{i} \frac{\pi}{2}-\Gamma(0,-\mathrm{i} z(1+\cos \theta))\right] \cos m \theta \mathrm{d} \theta \underset{z \rightarrow 0}{\longrightarrow}-\frac{2(-\mathrm{i})^{m}}{\pi|m|}
$$

and, therefore, the behavior for $z \sim 0$ is dominated by the sum term. In formula (3.11) we retain the term with $j=|m|$ and then, recalling that $\Gamma\left(\frac{1}{2}-|m|\right) \Gamma\left(\frac{1}{2}+\right.$ $|m|)=(-1)^{m} \pi$, we obtain:

$$
Y_{m}(z) \underset{z \rightarrow 0}{\sim}-\frac{\mathrm{i}^{(m-|m|)}}{\pi} \Gamma(|m|)\left(\frac{2}{z}\right)^{|m|} \quad(z \in \mathbb{C}, m \in \mathbb{Z}, m \neq 0),
$$

which coincides indeed with formula (3.28) when $\mu \rightarrow m \in \mathbb{Z} \backslash\{0\}$.

For the case $m=0$ we refer to representation (3.13). We recall that $\Gamma(0, w)$ coincides with the exponential integral $E_{1}(w)$ [7, Eq. 6.2.1] and, moreover, it can be written as [7, Eq. 6.6.2]:

$$
\Gamma(0, w)=-\gamma-\ln w-\sum_{k=1}^{\infty} \frac{(-w)^{k}}{k \cdot k !},
$$


where $\gamma$ denotes the Euler-Mascheroni constant. Considering that $e^{-\mathrm{i} z \cos \theta} \rightarrow 1$ for $z \rightarrow 0$, we see for the first term in (3.13): $\frac{2}{\pi^{2}} \int_{0}^{\pi} \mathrm{i} \frac{\pi}{2} e^{-\mathrm{i} z \cos \theta} \mathrm{d} \theta \rightarrow \mathrm{i}$ for $z \rightarrow 0$. For what concerns the second term in (3.13), we use the following definite integrals:

$$
\int_{0}^{\pi} \ln (-\mathrm{i} z(1+\cos \theta)) \mathrm{d} \theta=\pi \ln (-\mathrm{i} z / 2), \quad \int_{0}^{\pi}(1+\cos \theta)^{k} \mathrm{~d} \theta=\frac{2^{k} \sqrt{\pi} \Gamma\left(k+\frac{1}{2}\right)}{\Gamma(k+1)},
$$

and formula (3.31) to obtain:

$$
-\frac{2}{\pi^{2}} \int_{0}^{\pi} e^{-\mathrm{i} z \cos \theta} \Gamma(0,-\mathrm{i} z(1+\cos \theta)) \mathrm{d} \theta \underset{z \rightarrow 0}{\sim} \frac{2 \gamma}{\pi}+\frac{2}{\pi} \ln \left(\frac{z}{2 \mathrm{i}}\right)+\frac{4}{\pi} \sum_{k=1}^{\infty}\left(\frac{\mathrm{i} z}{2}\right)^{k} \frac{\Gamma(2 k)}{(k !)^{3}} .
$$

Finally, from (3.13) we obtain the following approximation near $z=0$ :

$$
Y_{0}(z) \underset{z \rightarrow 0}{\sim} \frac{2}{\pi}[\ln (z / 2)+\gamma]+\frac{4}{\pi} \sum_{k=1}^{\infty}\left(\frac{\mathrm{i} z}{2}\right)^{k} \frac{\Gamma(2 k)}{(k !)^{3}} \underset{z \rightarrow 0}{\sim} \frac{2}{\pi}[\ln (z / 2)+\gamma] .
$$

\section{Representation of Hankel's functions $H_{\mu}^{(1,2)}(z)$}

Similarly to what we have done for the Neumann function $Y_{\mu}(z)$, a mixed-type representation, i.e., integral plus finite sum, can be obtained also for the Hankel functions of the first and second kind (also known as Bessel's functions of the third kind) $H_{\mu}^{(1)}(z)$ and $H_{\mu}^{(2)}(z)$ by using the formulae [7, Eqs. 10.4.7, 10.4.8] (see also (1.5)):

$$
\begin{aligned}
& H_{\mu}^{(1)}(z)=\mathrm{i} \frac{e^{-\mathrm{i} \mu \pi} J_{\mu}(z)-J_{-\mu}(z)}{\sin \mu \pi}, \\
& H_{\mu}^{(2)}(z)=-\mathrm{i} \frac{e^{\mathrm{i} \mu \pi} J_{\mu}(z)-J_{-\mu}(z)}{\sin \mu \pi} .
\end{aligned}
$$

Here, we limit ourselves to present the final representations since the procedure we follow is the same as the one presented in Section 3 for the Neumann functions. From (4.1) and (2.22) we have:

$$
-\mathrm{i} H_{\mu}^{(1)}(z)=\mathcal{H}_{\mu}^{(1)}(z)+T_{-}(\mu) \frac{\chi_{[-\mu \operatorname{sgn}(\operatorname{Re} \mu)]}}{\sin \mu \pi} \quad(\mu \in \mathbb{C}),
$$

where:

$$
T_{-}(\mu) \doteq\left\{\begin{array}{lll}
e^{-\mathrm{i} \mu \pi} & \text { if } & \operatorname{Re} \mu<0, \\
-1 & \text { if } & \operatorname{Re} \mu \geqslant 0,
\end{array}\right.
$$

the integral term being:

$$
\begin{aligned}
& \mathcal{H}_{\mu}^{(1)}(z) \doteq \frac{e^{-\mathrm{i} \mu \pi} B_{\mu}(z)-B_{-\mu}(z)}{\sin \mu \pi} \\
& =\frac{\mathrm{i}^{-\mu}}{\pi \sin \mu \pi} \int_{0}^{\pi} e^{-\mathrm{i} z \cos \theta}[P(\{\mu\},-\mathrm{i} z(1+\cos \theta))-P(-\{\mu\},-\mathrm{i} z(1+\cos \theta))] \cos \mu \theta \mathrm{d} \theta .
\end{aligned}
$$


When $\mu \equiv m \in \mathbb{Z}, H_{m}^{(1)}(z)$ can be obtained as the limit for $\mu \rightarrow m$ of formula (4.5) and keeping into account the limit (3.5). We have (see also (3.14)):

$$
H_{m}^{(1)}(z)=\frac{2 \mathrm{i}^{m-1}}{\pi}\left[\frac{1}{\pi} \int_{0}^{\pi} e^{-\mathrm{i} z \cos \theta} \Gamma(0,-\mathrm{i} z(1+\cos \theta)) \cos m \theta \mathrm{d} \theta+\sigma_{m}(z)\right]
$$

where $\sigma_{m}(z)$ is the finite sum defined in (3.12).

Similarly, the representation for the Hankel function $H_{\mu}^{(2)}(z)$ follows from (4.2) and (2.22):

$$
\mathrm{i} H_{\mu}^{(2)}(z)=\mathcal{H}_{\mu}^{(2)}(z)+T_{+}(\mu) \frac{\chi_{[-\mu \operatorname{sgn}(\operatorname{Re} \mu)]}(z)}{\sin \mu \pi} \quad(\mu \in \mathbb{C}),
$$

where:

$$
T_{+}(\mu) \doteq\left\{\begin{array}{lll}
e^{\mathrm{i} \mu \pi} & \text { if } & \operatorname{Re} \mu<0 \\
-1 & \text { if } & \operatorname{Re} \mu \geqslant 0
\end{array}\right.
$$

the integral term being:

$$
\begin{aligned}
\mathcal{H}_{\mu}^{(2)}(z) \doteq & \frac{e^{\mathrm{i} \mu \pi} B_{\mu}(z)-B_{-\mu}(z)}{\sin \mu \pi}=\frac{\mathrm{i}^{\mu}}{\pi \sin \mu \pi} \int_{0}^{\pi} e^{-\mathrm{i} z \cos \theta} \\
& \cdot\left[e^{\mathrm{i} \mu \pi} P(\{\mu\},-\mathrm{i} z(1+\cos \theta))-e^{-\mathrm{i} \mu \pi} P(-\{\mu\},-\mathrm{i} z(1+\cos \theta))\right] \cos \mu \theta \mathrm{d} \theta .
\end{aligned}
$$

When $\mu \equiv m \in \mathbb{Z}$, we have:

$$
H_{m}^{(2)}(z)=-\frac{2 \mathrm{i}^{m-1}}{\pi}\left[\frac{1}{\pi} \int_{0}^{\pi} e^{-\mathrm{i} z \cos \theta}[\Gamma(0,-\mathrm{i} z(1+\cos \theta))-\mathrm{i} \pi] \cos m \theta \mathrm{d} \theta+\sigma_{m}(z)\right] .
$$

\section{REFERENCES}

1. M. Abramowitz, I.A. Stegun, Handbook of Mathematical Functions, 10th GPO Printing, Dover, New York (1972).

2. E. De Micheli, Integral representation for Bessel's functions of the first kind and Neumann series, Results Math. 73:61 (2018).

3. A. Erdelyi, W. Magnus, F. Oberhettinger, F.G. Tricomi, Higher Transcendental Functions, Vol. II, McGraw-Hill, New York (1953).

4. Gautschi, W., The incomplete gamma functions since Tricomi. In: Tricomi's Ideas and Contemporary Applied Mathematics, Atti dei Convegni Lincei 147, pp.203-237. Accademia Nazionale dei Lincei, Roma (1998)

5. I.S. Gradshteyn, I.M. Ryzhik, Table of Integrals, Series and Products, 7th Edition, Elsevier, Amsterdam (2007).

6. Y. L. Luke, The Special Functions and their Approximations, Vol. II, Academic Press, New York, (1969).

7. NIST Digital Library of Mathematical Functions. http://dlmf.nist.gov/ Release 1.0.15. F.W.J. Olver, A.B. Olde Daalhuis, D.W. Lozier, B.I. Schneider, R.F. Boisvert, C.W. Clark, B.R. Miller, and B.V. Saunders, eds.

8. Tricomi, F.G.: Asymptotische Eigenschaften der unvollständigen Gammafunktion. Math. Z. 53(2), 136-148 (1950)

9. Tricomi, F.G.: Sulla funzione gamma incompleta. Ann. Math. Pura Appl. 31(1), 263-279 (1950)

10. G.N. Watson, A Treatise on the Theory of Bessel Functions, Cambridge University Press, Cambridge, 1922.

Consiglio Nazionale delle Ricerche, Via De Marini, 6 - 16149 Genova, Italy

E-mail address: enrico.demicheli@cnr.it 